\title{
Tension Free Monofilament Macropore Polypropylene Mesh (Gynemesh PS) in Female Genital Prolapse Repair
}

\author{
Vicente Sola, Jack Pardo, Paolo Ricci, Enrique Guiloff \\ Urogynecology and Vaginal Surgery Unit, Gynecology and Obstetrics Department, Las Condes \\ Clinic, Chile University Clinical Campus, Santiago, Chile
}

\begin{abstract}
Objectives: To review intraoperative and postoperative complications associated to the correction of cystocele and rectocele with polypropylene mesh macropore monofilament (Gynemesh PS) using transvaginal free tension technique.

Materials and Methods: Prospective study of patients that have been submitted to correction of cystocele and/or rectocele between November 2004 and August 2005 in the Urogynecology and Vaginal Surgery Unit of Gynecology and Obstetrics Department, Las Condes Clinic. Mesh was used in 31 patients: 9 for cystocele, 11 for rectocele, and 11 for concomitant meshes. Total mesh used 42. Media age 55 years old, weight 64 kilograms. In 7 patients we used a third mesh for correction of urinary incontinence by TVT-O technique.

Results: They did not present intraoperative complications, neither in immediate or delayed postoperative time. We did not observe hematoma, infection, erosion or exposition mesh. Healing of cystocele and rectocele was obtained in $100 \%$ of patients, with a pursuit between 1 and 8 months.

Discussion: The use of prosthetic polypropylene monofilament macropore mesh in the correction of cystocele and/or rectocele, by transvaginal route with tension free technique seems to be a safe and effective surgery procedure.
\end{abstract}

Key words: cystocele, rectocele, polypropylene marlex

Int Braz J Urol. 2006; 32: 410-5

\section{INTRODUCTION}

Genital prolapse or genital hernia is known by the displacement of pelvic organs along the vagina. It is one of the pathological diseases that frequently affect the quality of life in women. It may be present in up to $50 \%$ of multipara women (1), and its incidence increases with age (2). High percentages of recurrence with traditional techniques (3), 2 to $40 \%$ for cystocele $(4,5)$, induced physiopathology of prolapse to be reconsidered and provoked the development of new surgical techniques. Meshes have, there- fore, been used in cystocele and rectocele repair to reduce recurrence, as well as experience in surgical repair of abdominal wall defects, such as inguinal and incisional hernias, among others. When choosing the mesh to be used, its properties become the key factor to achieve optimum results. Recently, more flexible and pliant meshes have been manufactured, such as polypropylene monofilament meshes. Another characteristic to be taken into account is the pore size, which is related to the risk of infection $(6,7)$.

We have initiated a prospective follow-up with 31 patients aiming at understanding the compli- 
cations associated to intraoperative and postoperative period in the use of monofilament macropore polypropylene mesh, using the tension free technique as reinforcement for the correction of cystocele and/ or rectocele.

\section{MATERIALS AND METHODS}

Prospective follow-up of patients hospitalized in the Urogynecology and Vaginal Surgery Unit of Gynecology and Obstetrics Department of Las Condes Clinic from November 2004 to June 2005 for the correction of cystocele and rectocele using monofilament macropore polypropylene mesh. All patients had symptomatic cystocele and or rectocele (sensation of mass or bulge in the vagina, pain, dispareunia, defecatory dysfunction, inability to evacuate the rectum and constipation). Patients with asymptomatic and mild degree prolapses were excluded. Prolapse degree was classified before the surgery according to the Baden and Walker graduation, with pelvic examination findings.

Ethicon Gynemesh PS (non-absorbable synthetic prosthesis) mesh, Johnson \& Johnson, has been used as reinforcement in the cystocele and/or rectocele correction, using transvaginal tension free technique.

\section{Surgical Technique}

Cystocele - Inverted-T incision under contact YAG Laser (10 watts) in vaginal mucous up to the level of vesical neck; dissection of vaginovesical space using Yag Laser, and fascia traction with a specialty sponge. Deep paravaginal dissection in digital roman-shape. This maneuver was made with the fingers in order to avoid dissection or damage of the fascia. Repair of prolapse, by closing in situ defects that are specific of the fascia of this site using vicryl 3/0. Cut out pieces of Gynemesh PS with scissors, leaving two tabs on each side. Place the mesh, positioning the tabs in paravaginal spaces, tension free, without stitches (Figure-1). Resection of vaginal mucous in approximately $5 \mathrm{~mm}$ on each side. Closure of vaginal mucous using continuous vicryl 3/0.
Rectocele - Typical inverted-T incision under contact Yag Laser (10 watts) in vaginal mucous up to 2 to $3 \mathrm{~cm}$ of the posterior cul-de-sac. Dissection of vagino-rectal space using Yag Laser, and fascia traction with a specialty sponge. Repair of prolapse, by closing in situ defects that are specific of the fascia of this site using vicryl 3/0. Cut out pieces of Gynemesh PS mesh with scissors. Tension free placement of mesh with 4 suture stitches of polypropylene $3 / 0$ on each edge, which are attached to the same recto-vaginal fascia previously corrected (Figure-2). Resection of vaginal mucous of

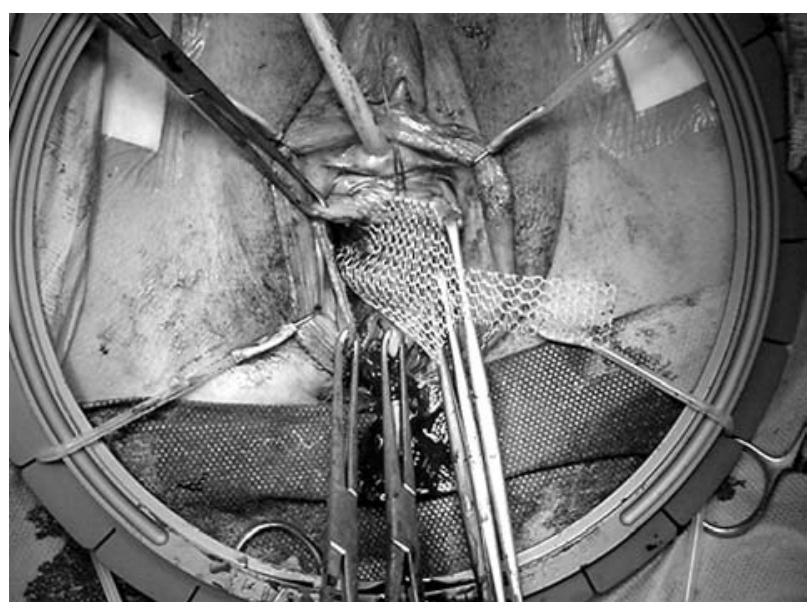

Figure 1-Surgical technique of cistocele repair with mesh. Mesh placement, positioning the tabs in paravaginal spaces, tension free, without stitches.

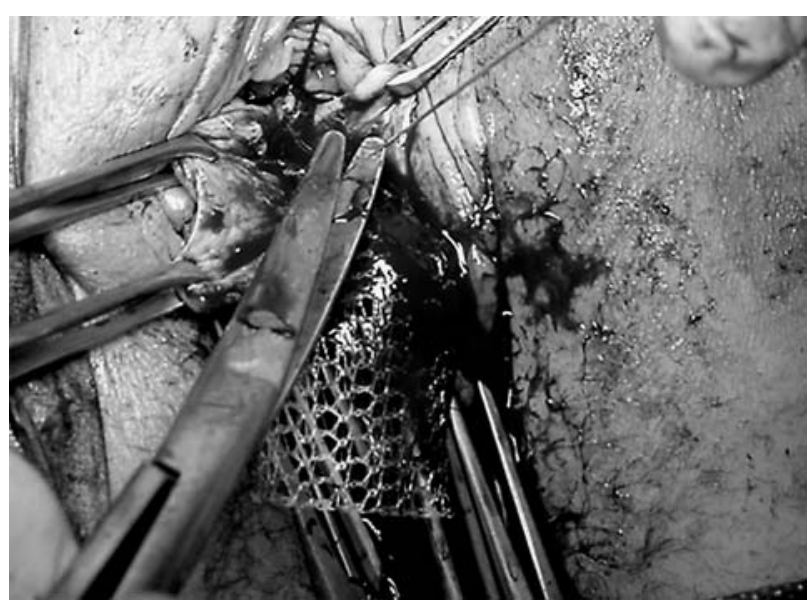

Figure 2 - Surgical technique of cistocele repair with mesh. Tension free placement of mesh with 4 suture stitches of polypropylene $3 / 0$ on each edge, which are attached to the same rectovaginal fascia previously corrected. 
$5 \mathrm{~mm}$ approximately on each side. Closure of vaginal mucous using continuous vicryl $3 / 0$.

In cases with paravaginal or pararectal defect, a site-specific repair of fascial defects to close them was made. Then, the edge of the fascial defects or tears was identified; the defect was repaired with interrumped and absorbable suture. The mesh tabs must be placed in the paravaginal or pararectal space without perforating fascia. All patients received spinal anesthesia.

Pelvic examination was performed in ambulatory control every week and soon every month to verify that the results were kept. In addition, patients were asked about the symptoms of the previous surgery and the new appearance, quality of life and acceptance.

Mesh was used in 31 patients: 9 for cystocele, 11 for rectocele, and 11 for concomitant pathologies. Total mesh used was 42 . Medium age 55 years old, weight 64 kilograms. All patients except one were in menopause, 28 under hormonal treatment for at least one year. Vaginal childbirth rank 1 to 5 , average 3 .

Five cases presented antecedent of surgery for prolapse correction (cistocele) with perineorrhaphy without mesh, in rank of 5 to 15 years.

In 7 patients, who received anterior and posterior mesh, a third mesh was also applied to correct an urinary incontinence by TVT-O technique (tension free transvaginal tape for the obturator hole, Johnson \& Johnson) (8).

During the surgery, antibiotic prophylaxis was delivered via a single intravenous dose of cefazoline of $2 \mathrm{~g}$.

As oral analgesia, valdecoxib $50 \mathrm{mg}$ was used one hour before the surgery, and a single oral dose was repeated every 24 hours for three days. Additionally, ketoprophene $100 \mathrm{mg}$ endovenous was administered every 8 hours only on the first day (three doses).

All patients were informed about the surgical procedure they would be submitted to. They all signed an informed consent. A data base for prospective pursuit was made.

The company supplier of the mesh did not warned about this study, in order to keep this experience in our patients free from contributions of mesh manufacturers.

\section{RESULTS}

Patients have cystocele grade 2 to 3 ; rectocele was mild grade. Seven events that showed urinary stress incontinence corresponded to type II.

The complete and maintained correction of cystocele and/or rectocele was verified in all ambulatory controls.

Average time of surgery was 19 minutes for cystocele and also 19 minutes for rectocele. There was no complication related to the placement of mesh for the correction of cystocele, or rectocele. Neither there was any complication related to the TVT-O, in the 7 events in which the technique was used. There was no complication related to anesthesia.

There was no immediate postoperative complications (up to 7 days) recorded. No hematoma or infection was observed in the operative area.

It was not required to involve additional pharmacotherapy to the plan originally developed to control postoperative pain.

All patients got up from hospital bed between 12 and 24 hours. Feed was introduced between 2 and 4 hours after surgery. Hospital discharge occurred 48 hours after surgery.

To date, after completing 1 to 8 month followup, no complication was found. No patient showed externalization of the mesh or vaginal erosion.

In all patients presenting sensation of mass or bulge before surgery, these symptoms disappeared. In cases of symptomatic rectocele it improved the rectum evacuation. No patients presented a new dyspareunia or vaginal discharge after procedure. Patients were satisfied with the results obtained. Those patients who maintained sexual relations before surgery, continued later without variation. The rest did not have sexual pair. All obtained cure (no episodes of incontinence) without increased complications.

\section{DISCUSSION}

The first study with polypropylene mesh for the correction of the prolapse of the anterior wall was conducted by Julian in 1996 using Marlex (5). Twenty four patients were randomized, 12 with correction 
using mesh. All patients that received the mesh showed an anatomical correction within 24 month against $66 \%$ of patients showing anterior colporrhaphy only. In a 2000 publication, Migliari (9) shows results of $100 \%$ healing in a 20.5 month follow-up for 12 patients with correction of cystocele using Prolene. Likewise, in other publications the benefits of decreasing prolapses recurrence was established, using reinforcement prosthetic meshes for the repair of cystocele, or rectocele.

However, when choosing an inappropriate mesh we can expect morbidity, as a result of greater chance of infection and erosion of vaginal wall (1013). In the same mentioned Julian's study, the healing was achieved in $100 \%$ patients with mesh, but $25 \%$ of related morbidity was added due to the features of the prosthetic material used. To prevent these complications, we should consider the pore sizes, which along with material and filament knit provide the level of flexibility to the mesh (14), decreasing the chance of erosion as it is larger.

Pores must have a size that allows leucocytes ( 9 to $15 \mu$ ) and macrophages ( 16 to $20 \mu$ ) transit to reduce the risk of infection (7). Multifilament meshes have filament spaces that are smaller than $10 \mu$, allowing transit of bacteria $(1 \mu)$, but not allowing macrophages and leucocytes transit, which facilitates the infection. All of this allows us to affirm that the best mesh would be the macropore mesh (pores larger than 75 microns) and monofilament.

Polypropylene meshes correspond to Type 1, according to the classification of surgical prosthesis (15), in other words, monofilament with macropore $(75 \mu)$, which meets the requirements above. We suggest that the use of tension free vaginal mesh should be an important contribution to the technique for the correction of pelvic floor defects, as it has been the use of reinforcement meshes in the repair of abdominal hernias.

Another measure that may reduce the chances of infection is the intraoperative use of antibiotics as prophylaxis; a measure that is part of our working protocol.

In other publications about Gynemesh PS, the erosion of vaginal wall though more reduced than with the use of other meshes is among $3.8 \%$ (12) and $7.5 \%$
(11). All 31 patients (42 meshes: 20 for cystocele, and 22 for rectocele) did not show this complication after the follow-up of an average of 6 months for cystocele, and 5 months for rectocele (maximum followup of 8 months). Adhoute (12) reports events with effective results of correction of $95 \%$ for cystocele, and $100 \%$ for rectocele using Gynemesh, in a follow-up of 27 months.

Regarding surgery time, Bader (11) described 20 minutes +/- 2.2 (16-24) in the time used for the technique for cystocele, similar to the time of our experiment. However, there is no publication reporting the time used to the technique applied for rectocele.

We point out 11 events with concomitant repair of cystocele and rectocele, but fewer publications reporting these characteristics (12). In other publications about correction with polypropylene monofilament macropore Gynemesh PS mesh, only cystocele was mentioned $(10,11)$. Dweyer (13) published his experience in events of cystocele correction whether related to rectocele or not, using polypropylene monofilament Atrium (Hudson, New Hampshire, USA) mesh.

In addition, we point out 7 events with three meshes, i.e., the use of the meshes combined with a TVT-O procedure with the typical technique for the correction of urinary stress incontinence, which did not mean increase of complications. This has proved that a combination of meshes can be safely and equally effective in the correction of pelvic floor defects and urinary incontinence.

All this let us think that the good results in terms of complications must have been due to the physical characteristics of this new mesh. We concluded that four mesh properties promote successful integration and this combination should be emphasized: polypropylene, elastic, macropore and monofilament mesh.

In cases with TVT-O for IUS corrections can be combined with mesh prolapse surgery, without increased complications. We put the TVT-O mesh through a different and separate vagina wall incision under the urethra. We let an undamaged mucous bridge ( 5 to $10 \mathrm{~mm}$ ) to avoid moving the tape out of this place. Previous distant incision for cistocele correction begins between 5 to $10 \mathrm{~mm}$. 
The results allow us to state that the use of tension free meshes, by vaginal route for the correction of cystocele and/or rectocele is an effective and safe procedure, as it did not present intraoperative complications, in neither immediate postoperative time, meeting the purposes suggested originally. In addition, there was no complication during followup and to date. A future publication will have the purpose of measuring long-term prolapse recurrence.

\section{CONFLICT OF INTEREST}

None declared.

\section{REFERENCES}

1. Carey MP, Dwyer PL: Genital prolapse: vaginal versus abdominal route of repair. Curr Opin Obstet Gynecol. 2001; 13: 499-505.

2. MacLennan AH, Taylor AW, Wilson DH, Wilson D: The prevalence of pelvic floor disorders and their relationship to gender, age, parity and mode of delivery. BJOG. 2000; 107: 1460-70.

3. Porges RF, Smilen SW: Long-term analysis of the surgical management of pelvic support defects. Am J Obstet Gynecol. 1994; 171: 1518-26; discussion 15268.

4. Lucente V, Hale D, Miller D, Madigan J: A Clinical Assesment of Gynemesh PS for the repair of pelvis organ prolapse (POP). Journal of pelvic medicine \& Surgery. 2004; 10 Suppl 1: S35.

5. Julian TM: The efficacy of Marlex mesh in the repair of severe, recurrent vaginal prolapse of the anterior midvaginal wall. Am J Obstet Gynecol. 1996; 175: 1472-5.

6. Deval B, Haab F: What's new in prolapse surgery? Curr Opin Urol. 2003; 13: 315-23.

7. Brun JL, Bordenave L, Lefebvre F, Bareille R, Barbie C, Rouais F, et al.: Physical and biological characteristics of the main biomaterials used in pelvic surgery. Biomed Mater Eng. 1992; 2: 203-25.

8. de Leval J: Novel surgical technique for the treatment of female stress urinary incontinence: transobturator vaginal tape inside-out. Eur Urol. 2003; 44: 724-30.

9. Migliari R, De Angelis M, Madeddu G, Verdacchi T: Tension-free vaginal mesh repair for anterior vaginal wall prolapse. Eur Urol. 2000; 38: 151-5.

10. De Tayrac R, Gervaise A, Fernandez H: Cystocele repair by the vaginal route with a tension-free sub-bladder prosthesis. J Gynecol Obstet Biol Reprod (Paris). 2002; 31: 597-9.

11. Bader G, Fauconnier A, Roger N, Heitz D, Ville Y: Cystocele repair by vaginal approach with a tensionfree transversal polypropylene mesh. Technique and results. Gynecol Obstet Fertil. 2004; 32: 280-4.

12. Adhoute F, Soyeur L, Pariente JL, Le Guillou M, Ferriere JM: Use of transvaginal polypropylene mesh (Gynemesh) for the treatment of pelvic floor disorders in women. Prospective study in 52 patients. Prog Urol. 2004; 14: 192-6.

13. Dwyer PL, O'Reilly BA: Transvaginal repair of anterior and posterior compartment prolapse with Atrium polypropylene mesh. BJOG. 2004; 111: 831-6.

14. Birch C, Fynes MM: The role of synthetic and biological prostheses in reconstructive pelvic floor surgery. Curr Opin Obstet Gynecol. 2002; 14: 527-35.

15. Chu CC, Welch L: Characterization of morphologic and mechanical properties of surgical mesh fabrics. $\mathrm{J}$ Biomed Mater Res. 1985; 19: 903-16.

\author{
Correspondence address: \\ Dr. Vicente Solà Dalenz \\ Las Condes Clinic \\ Lo Fontecilla 441, Las Condes \\ Santiago, Chile \\ Fax: + 562 210-4195 \\ E-mail:vsola@vtr.net
}




\section{Gynemesh PS in Female Genital Prolapse Repair}

\section{EDITORIAL COMMENT}

It is well documented that many minimallyinvasive procedures for stress incontinence are not harmless, innocuous, or complication free. It has become clear to me through my surgical sling experience that those synthetic meshes with the most favorable biomechanical properties and greatest likelihood of biocompatibility and successful integration are elastic, macro-pored, monofilament polypropylene.

Potential mesh complications when used in prolapse surgery may be magnified because of the larger sheets of mesh required, as opposed to the narrow tapes used for slings. The authors of this paper have corroborated that the same inherent mesh characteristics that mitigate incorporation issues in stress incontinence surgery are equally important in mitigating incorporation problems in augmented reconstructions for cystoceles and rectoceles. Long-term follow-up is critical, but the short-term results are well worthy of dissemination.

Dr. Andrew L. Siegel

Department of Urology

University of Medicine of New Jersey

Hackensack, New Jersey, USA 